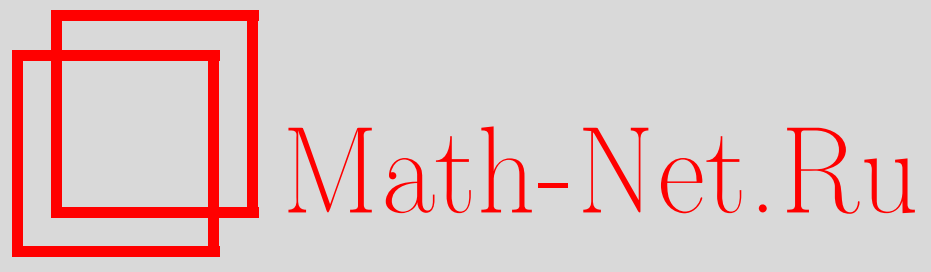

И. В. Вьюгин, Р. Р. Гонцов, К вопросу о разрешимости в квадратурах фуксовых систем, УМН, 2012, том 67, выпуск $3,183-184$

DOI: https://doi.org/10.4213/rm9438

Использование Общероссийского математического портала Math-Net.Ru подразумевает, что вы прочитали и согласны с пользовательским соглашением http://www . mathnet.ru/rus/agreement

Параметры загрузки:

IP : 54.198 .187 .58

26 апреля 2023 г., 15:28:12

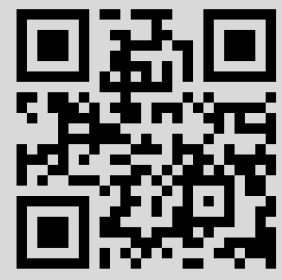




\section{К вопросу о разрешимости в квадратурах фуксовых систем}

\section{И. В. Вьюгин, Р. Р. Гонцов}

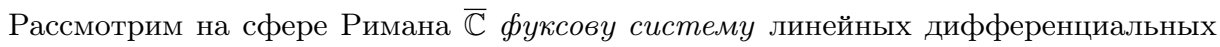
уравнений, т. е. систем вида

$$
\frac{d y}{d z}=\left(\sum_{i=1}^{n} \frac{B_{i}}{z-a_{i}}\right) y,
$$

где $y(z)$ - вектор в $\mathbb{C}^{p}, B_{1}, \ldots, B_{n}$ - комплексные $(p \times p)$-матрицы (матрицы-вычеты системы в соответствующих особых точках $\left.a_{1}, \ldots, a_{n} \in \mathbb{C}\right)$. Говорят, что она разрешима в квадратурах, если любое решение содержится в некотором расширении $F$ поля $\mathbb{C}(z)$ рациональных функций, полученном при помощи присоединения экспонент и интегралов: $\mathbb{C}(z)=F_{1} \subseteq \cdots \subseteq F_{m}=F$, где поле $F_{i+1}=F_{i}\left\langle x_{i}\right\rangle$ получается присоединением к полю $F_{i}$ элемента $x_{i}$, являющегося экспонентой или интегралом некоторого элемента поля $F_{i}$.

Необходимым и достаточным условием разрешимости в квадратурах фуксовой системы (1) является разрешимость ее группы монодромии, т. е. группы, порожденной матрицами монодромии $G_{1}, \ldots, G_{n}$ в особых точках $a_{1}, \ldots, a_{n}$ (см. [1; гл. 6 , теорема 1.12]). В случае когда коэффициенты $B_{i}$ фуксовой системы (1) малы, Ю. С. Ильяшенко и А. Г. Хованский [2] получили явный критерий разрешимости такой системы.

Существует $\varepsilon=\varepsilon(n, p)>0$ такое, что условие разрешимости в квадратурах для фуксовой системы (1) $c\left\|B_{i}\right\|<\varepsilon$ принимает явный вид: система решается в квадратурах, если и только если все матрицы $B_{i}$ (в некотором базисе) треугольны.

Заметим, что в доказательстве данного утверждения авторы использовали соображения И. А. Лаппо-Данилевского [3], относящиеся к проблеме Римана-Гильберта при близости матриц монодромии к единичной. В книге [1] (с. 221) А. Г. Хованский указывает на предположение А. А. Болибруха о том, что в условии критерия разрешимости требование малости матриц $B_{i}$ можно ослабить. Достаточно лишь требовать, чтобы собственные числа этих матрии, были малы. В следующей теореме мы уточняем формулировку этого предположения и приводим его доказательство.

Теорема 1. Пусть собственные значения $\beta_{i}^{j}$ матрии $B_{i}$ таковы, что

$$
\operatorname{Re} \beta_{i}^{j}>-\frac{1}{n(p-1)}, \quad i=1, \ldots, n, \quad j=1, \ldots, p,
$$

и для каждой пары $\beta_{i}^{j} \neq \beta_{i}^{k}(i=1, \ldots, n)$ выполнено одно из двух условий:

$$
\text { 1) } \left.\operatorname{Re} \beta_{i}^{j}-\operatorname{Re} \beta_{i}^{k} \notin \mathbb{Q} ; \quad 2\right) \operatorname{Im} \beta_{i}^{j} \neq \operatorname{Im} \beta_{i}^{k} .
$$

Тогда разрешимость фуксовой системь (1) в квадратурах эквивалентна треугольности (в некотором базисе) всех матрии, $B_{i}$.

Доказательство начнем с двух вспомогательных лемм.

Лемма 1. Пусть собственные значения $\beta_{i}^{j}$ матриц-вычетов $B_{i}$ удовлетворяют условию (2). Тогда представление монодромии такой системы верхнетреугольно, если и только если все матрицы $B_{i}$ (в некотором базисе) верхнетреуголъны.

ДокАзАтЕльство. Воспользуемся геометрической интерпретацией (подробно изложенной в книге А. А. Болибруха [4]), согласно которой фуксовой системе (1) соответствует голоморфно тривиальное векторное расслоение $E$ ранга $p$ над сферой Римана с логарифмической связностью $\nabla$. Поскольку представление монодромии системы

Работа выполнена при поддержке РФФИ (грант № 11-01-00339), программы Президента РФ по поддержке молодых российских ученых (грант МК-4270.2011.1) и программы "Ведущие научные школы" (грант НШ-5998.2012.1). 
верхнетреугольно, то существует $E^{1} \subset E^{2} \subset \cdots \subset E^{p}=E$ - полный флаг подрасслоений рангов $1,2, \ldots, p$ соответственно, которые стабилизируются связностью $\nabla$ (всякому подпредставлению монодромии размерности $k$ соответствует подрасслоение ранга $k$, стабилизирующееся связностью).

Степень голоморфного векторного расслоения с логарифмической связностью определяется как сумма всех собственных значений матриц-вычетов связности по всем особым точкам. Для того чтобы найти степень подрасслоения ранга $k$, которое стабилизируется связностью, нужно в каждой особой точке просуммировать некоторые $k$ собственных значений матрицы-вычета. Из условия леммы следует, что степени всех подрасслоений $E^{k}$ равны нулю. Действительно, $\operatorname{deg} E^{p}=0$, а для $k \leqslant p-1$ имеем:

$$
\operatorname{deg} E^{k}=\sum_{i=1}^{n} \sum_{j \in J_{i},\left|J_{i}\right|=k} \beta_{i}^{j}=\sum_{i=1}^{n} \sum_{j \in J_{i},\left|J_{i}\right|=k} \operatorname{Re} \beta_{i}^{j}>-\frac{k}{p-1} \geqslant-1 \Longrightarrow \operatorname{deg} E^{k}=0
$$

(степень подрасслоения голоморфно тривиального векторного расслоения не положительна). Таким образом, все подрасслоения $E^{k}$ голоморфно тривиальны (подрасслоение голоморфно тривиального векторного расслоения голоморфно тривиально, если его степень равна нулю), и, следовательно, матрицы $B_{i}$ одновременно приводятся к верхнетреугольному виду (см. [4; замечание 11.2]). Лемма доказана.

Матрицу $G$ назовем $N$-резонансной, если найдутся два ее собственных значения $\lambda_{1} \neq \lambda_{2}$ такие, что $\lambda_{1}^{N}=\lambda_{2}^{N}$, т. е. $\left|\lambda_{1}\right|=\left|\lambda_{2}\right|, \arg \lambda_{1}-\arg \lambda_{2}=\frac{2 \pi}{N} j, j \in\{1,2, \ldots, N-1\}$. Согласно замечанию после теоремы 2.7 в [1; гл. 6], справедлива следующая лемма.

Лемма 2. Пусть группа $H \subset \mathrm{GL}(p, \mathbb{C})$ порождена матрицами $G_{1}, \ldots, G_{n} . C y$ ществует число $N=N(p)$ такое, что если матрицы $G_{1}, \ldots, G_{n}$ не являются $N$-резонансными, то из разрешимости группы $H$ следует треугольность этих матрии.

ДоКАЗАТЕЛЬСТво теОРемы 1 . Собственные значения $\lambda_{i}^{j}$ матрицы монодромии $G_{i}$ связаны с собственными значениями $\beta_{i}^{j}$ матрицы-вычета $B_{i}$ фуксовой системы соотношением $\lambda_{i}^{j}=\exp \left(2 \pi \mathbf{i} \beta_{i}^{j}\right), \mathbf{i}=\sqrt{-1}$ (см. [4; лекция 6]). Таким образом,

$$
\lambda_{i}^{j}=\exp \left(2 \pi \mathbf{i}\left(\operatorname{Re} \beta_{i}^{j}+\mathbf{i} \operatorname{Im} \beta_{i}^{j}\right)\right)=e^{-2 \pi \operatorname{Im} \beta_{i}^{j}}\left(\cos \left(2 \pi \operatorname{Re} \beta_{i}^{j}\right)+\mathbf{i} \sin \left(2 \pi \operatorname{Re} \beta_{i}^{j}\right)\right),
$$

и в силу условий на числа $\beta_{i}^{j}$ матрицы $G_{i}$ не могут быть $N$-резонансными ни при каком $N$. Теперь утверждение теоремы следует из лемм 1,2 .

\section{Список литературы}

[1] А.Г. Хованский, Топологическая теория Галуа. Разрешимость и неразрешимость уравнений в конечном виде, МЦНМО, М., 2008, 296 с. [2] Ю. С. Ильяшенко, А. Г. Хованский, Теория Галуа систем дифференциалъных уравнений типа Фукса с малыми коэффициентами, Препринт ИПМ АН СССР № 117, 1974. [3] И. А. Лаппо-Данилевский, Применение функиий от матрии, $x$ теории линейных систем обыкновенных дифференциальных уравнений, ТехТеорЛит, М., 1957, 456 с. [4] А.А. Болибрух, Обратные задачи монодромии в аналитической теории дифференциальных уравнений, МЦНМО, М., 2009, 200 с.

И. В. Вьюгин (I. V. Vyugin)

Институт проблем передачи информации им. А. А. Харкевича РАН

E-mail: vyugin@gmail.com

\section{P. P. Гонцов (R. R. Gontsov)}

Институт проблем передачи информации им. А. А. Харкевича РАН

E-mail: gontsovrr@mpei.ru
Представлено Д. В. Аносовым Принято редколлегией 01.09.2011 